\title{
Occupational Health Hazards \& Efficacy of Protective Masks in Threshing Operation
}

\author{
Sudesh Gandhi ${ }^{\mathrm{a} 1}$, M. Dilbaghi ${ }^{\mathrm{b}}$, M. Mehta ${ }^{\mathrm{c}}$ and Neelam Pruthi ${ }^{\mathrm{d}}$ \\ ${ }^{a}$ Senoir Scientist Deptt. of Family Resource Management, COHS,CCSHAU,Hisar,Haryana,India. \\ ${ }^{b}$ Reseach Associate, Deptt. of Family Resource Management, COHS,CCSHAU,Hisar, Haryana,India. \\ ${ }^{c}$ Scientist, Deptt. of Family Resource Management, COHS,CCSHAU,Hisar, Haryana,India. \\ ${ }^{d}$ Senoir Scientist Deptt. of Textile and Apparel Designing, COHS,CCSHAU,Hisar, Haryana,India.
}

\begin{abstract}
The study was conducted on 20 farm workers to identify the occupational health hazards in threshing operation. Four different protective masks were given and tested on various parameters. Respondents reported respiratory health problems which were mainly due to heat and organic dust in the surroundings. All respondents reported irritation in eyes and throat followed by nose $(85.0 \%)$ and ears $(75.0 \%)$. Musculo-skeletal problems were reported showing severe to very severe discomfort in lower arms (m.s.=3.8), upper back (3.6) and upper arms (3.4). Overall discomfort score (ODS) was 7.6 depicting high level of discomfort. Peak expiratory flow rate was reduced by $6.6 \%$ depicting reduced capacity of lungs after day long work in polluted environment. Hood mask was highly acceptable as it showed lowest breathing resistance. Leakage of dust from sides and rate of sweating was medium. Hence, use of hood mask would be helpful to achieve the ergonomics objective of reducing health problems and improving performance.
\end{abstract}

Key words: peak flow rate, respiratory problems, breathing resistance, overall discomfort score

\section{Introduction}

Wheat being a major staple crop is grown in most part of India. Harvesting of wheat is commonly done in the month of March-April. After cutting crop, farmers hire the thresher and work continuously day and night to complete the threshing process. Agriculture, being a family occupation, most of the family members including women, are involved in performing this activity.

In harvesting season, farmers are exposed to high levels of organic dust during threshing of crops leading to many health hazards. Grain dust inhalation may induce respiratory diseases including chronic bronchitis, granulomatous pneumonitis and toxic pneumonitis which finally may lead to decreased lung function (Melbostad et al., 1997[2]; Swan and Crook, 1998[3]). With combine harvester, situation goes even much worse. As volume of dust generated is so high that even people living in nearby area are affected and breathing problem cases increase during the harvesting season. Studies reveal that respiratory diseases due to organic dust, dust mites, molds and other organic antigens, are also common in the developing countries during these kinds of activities that cause chronic health hazards to the workers.

No doubt farmers have been using indigenous techniques to protect themselves during threshing

\footnotetext{
${ }^{1}$ Corresponding author.

E-mail: Sggandhi@gmail.com,sgandhi@hau.ernet.in
} 
operation i.e. covering their face and head by using a cloth but its efficacy is very limited. Along with breathing problems, people working in such environment without well-protected clothing may lead to physical fatigue resulting in the reduction of work. Hence, protection from dust is very essential as it would reduce microbial exposure. In light of he above, different types of masks were designed and tested to alleviate the farmers from exposure to dust with the objectives: i) Studying the occupational health hazards in threshing activity ii) testing the efficacy of protective masks against health hazards.

\section{Methodolody}

The present study was carried out on 20 farm workers including men (10) and women (10) doing wheat threshing in the month of March-April, 2010. The experiment was conducted for one hour and their observations were recorded. Experiment was conducted in two phases-

Phase I- Occupational health hazards \& preventive measures

Under phase 1, data were collected on occupational health hazards of threshing operations studying their health problems, musculo-skeletal discomfort and overall discomfort score. Health problems of various body parts were recorded using observation sheet on 3 point continuum ranging from never (1) to mostly (3). Musculo- skeletal discomfort were studied using human body map to identify incidences of problems in different parts of the body [1] on a five point scale ranging from very severe pain (5) to very mild pain (1). For the assessment of overall discomfort rating, a psycho-physical rating scale at 10 point continuum, 0 being the lowest point showing no discomfort and 10 being the uppermost point showing extreme discomfort was used [1]. Various indigenous techniques adopted by rural people as precautions to protect themselves during threshing process were studied recording frequency of use on 3 point continuum i.e. always, sometimes and never.

Phase II- Efficacy testing of protective masks

Under phase II, four types of face masks viz beakmask, face mask, hood mask and scarf mask developed by Dept. of Textile and Apparel Designing, CCS HAU , Hisar were tested for its efficacy on three point continuum of performance viz. low (1), medium (2) and high (3).
These masks were used to protect the inhalation of organic dust as well as exposure of various body parts i.e. head, face, ears and neck. Various statements were prepared to test the suitability performance in terms of breathing resistance, dust filtration performance, leakage of air from sides, feeling of tightness on face, rate of sweating and acceptability on three point continuum scale. These were given ranks on the basis of scores. Peak flow master was used to measure the peak expiratory flow rate of the worker before and after the work.

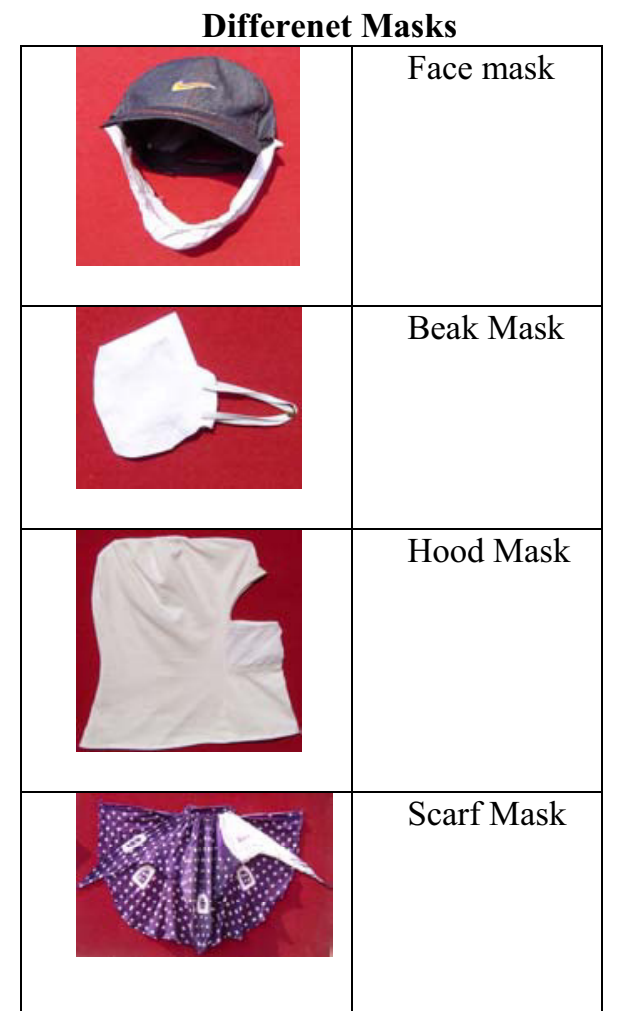

\section{Results and Discussions}

\subsection{Occupational health hazards and preventive measure}

Occupational health hazards during threshing revealed that health problems during threshing were mainly due to heat and dust (Table 1). This shows that mostly they suffered with irritation in eyes, throat, nose and ears affecting their respiratory tract. 
It was almost difficult to stand there for collecting data during the experiment.

Moreover, while working the temperature was 43.8 $\mathrm{C}$ that was making work environment more uncomfortable so heat stroke was also observed as hazards for many. So, this worsened the situation \& caused headache to majority of the respondents. Cuts and abrasions were also observed due to pricking of dry straws while collecting and lifting the crop bundles for feeding into chute of the thresher. Cent percent respondents reported irritation in eyes and throat followed by nose $(85.0 \%)$ and ears $(75.0 \%)$.

Table1. Health problems while wheat threshing

\begin{tabular}{|lllll|}
\hline \multicolumn{1}{|c}{ Problems } & Mostly & $\begin{array}{l}\text { Some- } \\
\text { times }\end{array}$ & Never & $\begin{array}{l}\text { Mean } \\
\text { score }\end{array}$ \\
\hline Eyes Irritation & $20(100.0)$ & - & & 3 \\
\hline Nose Irritation & $17(85.0)$ & $3(15.0)$ & & 2.9 \\
\hline $\begin{array}{l}\text { Throat } \\
\text { Irritation }\end{array}$ & $20(100.0)$ & - & & 3 \\
\hline Irritation in ear & $15(75.0)$ & $4(20.0)$ & $1(5.0)$ & 2.7 \\
\hline Headache & $10(50.0)$ & $6(30.0)$ & $1(20.0)$ & 2.2 \\
\hline $\begin{array}{l}\text { Breathing } \\
\text { problem }\end{array}$ & $2(10.0)$ & $8(40.0)$ & $10(50.0)$ & 1.5 \\
\hline Coughing & - & $3(15.0)$ & $17(85.0)$ & 1.2 \\
\hline Nausea & - & $4(20.0)$ & $16(80.0)$ & 1.2 \\
\hline $\begin{array}{l}\text { Hearing } \\
\text { difficulty }\end{array}$ & - & $5(25.0)$ & $15(75.0)$ & 1.3 \\
\hline Sunburns & $2(10.0)$ & $8(40.0)$ & $10(50.0)$ & 1.6 \\
\hline Heat stroke & $4(20.0)$ & $9(45.0)$ & $7(35.0)$ & 1.9 \\
\hline Cuts/Abrasion & - & $9(45.0)$ & $11(55.0)$ & 1.5 \\
\hline Skin allergies & $3(15.0)$ & $3(15.0)$ & $14(70.0)$ & 1.5 \\
\hline
\end{tabular}

Respondents reported these problems as common \& occurring mostly to the respondents during threshing. Half of the respondents reported headache mostly followed by sometimes $(30.0 \%)$. Occurrence of breathing problem was sometimes $(40.0 \%)$. Problems which were occurring sometimes were heat stroke (45.0\%), cuts and abrasion (45.0\%), sun burn (40.0\%), hearing problems (25.0) and nausea $(20.0 \%)$. Problems occurring mostly during threshing process were irritation in eyes, throat, nose and ear.

\subsubsection{Musculo-skeletal discomfort}

Body discomfort in different parts depicted that during threshing severe to very severe discomfort was in wrists, ankle/feet (4.7 each), hands (4.6) and shoulders (4.5). Moderate to severe discomfort was reported in lower arms (3.8), upper back ( 3.6) upper arms (3.4) and legs (3.4)(Fig 1). This was mainly due to the women were lifting and fetching the wheat bundles for collecting near the thresher site. They were handling over to the men standing near the feeding chute of the thresher. As they were lifting and fetching 12 bundles weighing about $22 \mathrm{~kg} / \mathrm{each}$ bundle during the experiment. While doing this activity, they adopted unnatural body postures comprising bending, lifting weight with shoulders and arms stretching upwards for holding bundle as head load \& releasing it by throwing this head load at the collection site of thresher. During the process, she puts strain on her wrists, ankles, feet, shoulders, arms, upper back \& neck leading to various musculo-

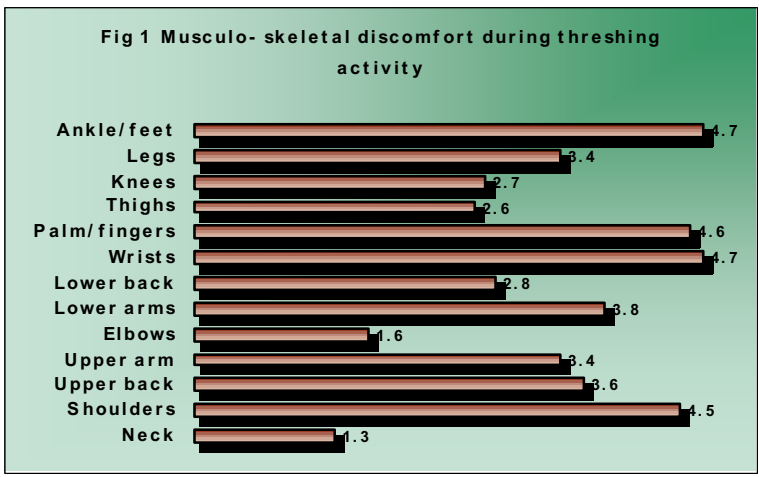

skeletal discomforts.

\subsubsection{Overall discomfort score}

Results reveal that overall discomfort score on 10 point scale was 7.6 for threshing activity interpreting that respondents felt this activity as very heavy activity \& they reported quite high level of discomfort during the activity.

\subsubsection{Prevention measures}

The preventive measures taken during threshing operations in table 2 reveal that majority of the respondents $(90.0 \%)$ covered their nose and mouth always by tying a piece of cloth during activity to protect themselves from the organic dust entering in their respiratory track. Majority of the respondents used to wear full sleeved clothing ( $85 \%$ ) followed by drinking plenty of water $(80 \%)$ while only 5 percent respondents used eye glasses sometimes to protect their eyes. Preventive measures were taken to protect themselves partially from dust and dirt but not the other health hazards. 
Table 2 Preventive measures taken while threshing operation

\begin{tabular}{|llll|}
\hline $\begin{array}{l}\text { Measures } \\
\text { taken }\end{array}$ & \multicolumn{1}{c}{ Always } & \multicolumn{1}{c}{ Sometime } & Never \\
\hline Cover head & $10(50.0)$ & $8(40.0)$ & $2(10.0)$ \\
\hline Cover neck & & $6(30.0)$ & $14(70.0)$ \\
\hline $\begin{array}{l}\text { Cover nose and } \\
\text { mouth }\end{array}$ & $18(90.0)$ & $2(10.0)$ & \\
\hline $\begin{array}{l}\text { Use full sleeved } \\
\text { clothing }\end{array}$ & $17(85.0)$ & $3(15.0)$ & \\
\hline
\end{tabular}

\subsubsection{Peak expiratory flow rate (PEFR)}

Peak expiratory flow rate before the activity was $241 \mathrm{~L} / \mathrm{min}$ which reduced to $225 \mathrm{~L} / \mathrm{min}$ after the activity. There was reduction of $16 \mathrm{~L} / \mathrm{min}$ in peak expiratory flow rate after day long activity. Percentage reduction in peak flow meter reading was $6.6 \%$ depicting reduced capacity of lungs after day long working in dust and sun.

\subsection{Efficacy testing of dust protectors}

Four different masks viz., beak mask, face mask, hood mask and scarf mask were given to rural women and were tested on various parameters.

Table 3. Performance testing of dust protectors(mean score)

\begin{tabular}{|lllll|}
\hline & $\begin{array}{c}\text { Beak } \\
\text { Mask }\end{array}$ & $\begin{array}{c}\text { Face } \\
\text { mask }\end{array}$ & $\begin{array}{c}\text { Hood } \\
\text { mask }\end{array}$ & $\begin{array}{c}\text { Scarf } \\
\text { mask }\end{array}$ \\
\hline Breathing resistance & 1.1 & 1.2 & 1.2 & 1.0 \\
\hline $\begin{array}{l}\text { Dust filtration perfor- } \\
\text { mance }\end{array}$ & 1.4 & 1.6 & 1.2 & 2.1 \\
\hline Comfortable during wear & 1.5 & 1.2 & 2.4 & 2.2 \\
\hline Rate of Sweating & 2.0 & 1.8 & 1.8 & 1.6 \\
\hline Acceptability & 2.2 & 2.3 & 2.8 & 2.4 \\
\hline
\end{tabular}

Table 3 reveals that breathing resistance was low (1-1.2) among the four mask interpreting that they could breathe easily in all the masks. Dust filtration was low in all the masks. It may be due to reason that masks were made of single layer of cloth which was not able to filter the air. Regarding feeling of tightness on face, beak mask and face mask were fine whereas hood mask and scarf mask were lights. Rate of sweating was medium (1.6 to 2.0) in all the masks. Regarding acceptability, beak mask face mask and scarf mask were medium acceptable whereas hood mask was highly acceptable. Regarding ranks hood mask scored $1^{\text {st }}$ rank followed by beak mask $\left(2^{\text {nd }}\right)$, face mask $\left(3^{\text {rd }}\right)$ and beak mask $\left(4^{\text {th }}\right)$.
Acceptability_Rank as per preference and acceptability

\begin{tabular}{|l|l|}
\hline Type of mask & Rank \\
\hline Beak-mask & $\mathbf{4}^{\text {th }}$ \\
\hline Face mask & $\mathbf{3}^{\text {rd }}$ \\
\hline Hood mask & $\mathbf{1}^{\text {st }}$ \\
\hline Scarf mask & $\mathbf{2}^{\text {nd }}$ \\
\hline
\end{tabular}

\section{References}

[1] Corlette, E.N. and Bishop, R.P. 1976. ' A technique for assessing postural discomfort, Ergonomics 19(2): 175-180.

[2] Melbostad, E., Eduard, W., \& Magnus, P. (1997). Chronic bronchitis in farmers. Scand J Work Environ Health, 23, 27180.

[3] Swan, J.R.M., \& Crook, B. (1998). Airborne microorganisms associated with grain handling. Ann Agric Environ Med, 5, $7-$ 15 